Pacific Journal of Mathematics

SINGULARITIES OF SUPERPOSITIONS OF DISTRIBUTIONS 


\title{
SINGULARITIES OF SUPERPOSITIONS OF DISTRIBUTIONS
}

\author{
DONALD LUDWIG
}

\section{Distributions of the form}

$$
F(x, \lambda)=\frac{1}{\Gamma\left(\frac{\lambda+1}{2}\right)} \int|f(x, u)|^{\lambda} g(x, u) d u
$$

are considered, where $x$ and $u$ belong to $R^{p}$ and $R^{n}$ respectively. The parameter $\lambda$ is complex, and $F(x, \lambda)$ is evaluated for $R e(\lambda)<0$ by analytic continuation. Such integrals arise in solution formulas for partial differential equations. In case $n=1$ or $n=2, F$ is expressed in terms of homogeneous distributions of degree $>\lambda+\alpha$, where $\alpha$ is nonnegative and depends upon the geometry of the roots of $f$. The case of general $n$ is also treated, in case the Hessian of $f$ with respect to $u$ is different from zero. The results lead to asymptotic expansions of analogous multiple integrals.

We assume that $f$ and $g$ are $C^{\infty}$ real-valued functions, and we assume that the gradient of $f$ with respect to $x$ does not vanish in the region of $R^{p} \times R^{n}$ under consideration. Integration is taken over a compact region $U \subset R^{n}$, and we assume that $g$ has its support in the interior of $U$. For $\operatorname{Re}(\lambda)>0$, the operation of $F$ on a test function $\varphi$ is defined by $I(\lambda)=\int F \varphi d x$. For other values of $\lambda, I(\lambda)$ is evaluated by an analytic continuation in $\lambda$. The factor $1 / \Gamma[(\lambda+1) / 2]$ ensures that $I(\lambda)$ is an entire function of $\lambda$. We actually require only a finite number of derivatives of $f$ and $g$, provided that $R e(\lambda)$ is bounded from below.

It is easy to see that, after a change of variables in $x$-space, $F_{1}\left(x_{1}, \lambda ; x_{2}, \cdots, x_{p}\right)=F\left(x_{1}, x_{2}, \cdots, x_{p}, \lambda\right)$ is a distribution in $x_{1}$, with $x_{2}, \cdots, x_{p}$ regarded as parameters. In case $n=1$ or $n=2$, we show that $F_{1}$ may be expressed as a sum of homogeneous distributions, plus a smooth remainder. Each term in the expansion of $F_{1}$ is associated with a point or points where $f(x, u)=0$ and $(\partial f / \partial u)(x, u)=0$. Expressions such as $(\partial f / \partial x)$ and $(\partial f / \partial u)$ denote the gradients with respect to the $x$ and $u$ variables, respectively. In case $n=1$, the most singular term of $F_{1}$ has the degree $\lambda+(1 / m)$, if $f$ has order $m$ with respect to $u$ at the corresponding point. In case $n=2$, the degree of the most singular term of $F_{1}$ depends upon the geometry of the real roots

Received February 6, 1964. This research was supported in part by National Science Foundation Grant G-22982. 
of $f$, regarded as functions of $\left(u_{1}, u_{2}\right)$ for fixed $x$. The degree of the singularity varies between $\lambda+(1 / m)$ and $\lambda+(2 / m)$, if $f$ has order $m$ with respect to $u$ at the point in question. The extreme values of the degree are assumed in case all roots of $f$ are coincident, or distinct, respectively. We also consider higher values of $n$, in the case where the Hessian matrix $\left(\partial^{2} f / \partial u_{i} \partial u_{j}\right)$ is nonsingular, which frequently arises in applications. In this case, the most singular part of $F$ is homogeneous of degree $\lambda+(n / 2)$.

Integrals of the form (1) arise in representations of solutions of hyperbolic partial differential equations, specifically the HerglotzPetrovsky formula and its generalizations. (See I. M. Gelfand and G. E. Shilov [7] pp. 137-141, and R. Courant [2], pp. 727-733.) We shall apply the results of the present paper to the analysis of the singularities of fundamental solutions of linear hyperbolic equations in a forthcoming revision of [10].

Our results also have implications for the asymptotic behavior of single and double integrals, using a device of D. S. Jones and M. Kline [8]. Let

$$
I(k)=\int \exp [i k f(u)] g(u) d u
$$

Then

$$
I(k)=\int e^{i k t} h(t) d t, \quad \text { where } h(t)=\int \delta(t-f(u)) g(u) d u .
$$

Here $\delta$ represents the one-dimensional Dirac function. The behavior of $I(k)$ for large $k$ is determined by the singularities of $h(t)$ (see A. Erdelyi [4], pp. 46-51.) But $h(t)$ is of the form (1), if we set $\lambda=-1$. For double integrals, our results extend those of D. S. Jones and M. Kline [8] and J. Focke [5] to give asymptotic expansions in cases where all derivatives of $f$ of second order vanish at some point.

The outline of our work is as follows: the first section is devoted to preliminary remarks, which apply for any $n$. We show that $F$ is a distribution in a single variable, and that singularities of $F_{1}$ at $x_{0}$ are associated with points $u$ where $f\left(x_{0}, u\right)=0$ and $(\partial f / \partial u)\left(x_{0}, u\right)=0$. In the second section, we reduce the case $n=1$ to consideration of an integral of the form

$$
I(x, \lambda, \alpha)=\gamma(\lambda) \int_{0}^{a}|x+u|^{\lambda} u^{\alpha-1} d u,
$$

where $\alpha$ is a real number. Here and henceforth, we write $\gamma(\lambda)=$ $1 / \Gamma[(\lambda+1) / 2]$. We analyze the singularities of (2) for arbitrary complex $\lambda$, and for $\operatorname{Re}(\alpha)>0$, using analytic continuation in both $\lambda$ and $\alpha$. The result is that $I(x, \lambda, \alpha)$ is the sum of a homogeneous distri- 
bution of degree $\lambda+\alpha$, and a smooth function. In the third section, we consider double integrals. We resolve the singularities of the zeros of $f$ by a series of quadratic transformations, and reduce the problem to consideration of integrals of the form

$$
I(x, \lambda, \alpha, \beta)=\gamma(\lambda) \iint\left|x+u^{\alpha} v^{\beta}\right| \lambda u^{\gamma-1} v^{\delta-1} g(u, v, \lambda) d u d v .
$$

In the fourth section, we expand (3) in powers of $x$. The integral is reduced to the form (2), or

$$
I^{\prime}(x, \lambda, \alpha)=\gamma(\lambda) \int_{0}^{a}(x+u)^{\lambda} u^{\alpha-1} \log u d u .
$$

$I^{\prime}(x, \lambda, \alpha)$ is just the derivative of $I(x, \lambda, \alpha)$ with respect to $\alpha$. The fifth section is devoted to the simpler case of integrals where the Hessian of $f$ with respect to $u$ does not vanish. In this case, the leading singularity of $F$ has degree $\lambda+(n / 2)$.

Our procedures, especially in the case of double integrals, would be rather unwieldy for purposes of calculation. A simpler scheme is presented by G. F. D. Duff [3]. Our results may be regarded as a justification of certain of his methods. Our methods and results, especially in $\S \S 2$ and 5 , have much in common with L. Gårding [6].

1. General remarks. In this section, we shall first show that integrals of the form (1) define distributions in a single variable, with smooth (in distribution sense) dependence on the other variables as parameters. Then we show that the singularities of such integrals are associated with points where $f$ and $\partial f / \partial u$ both vanish. This fact is the analog of the principle of stationary phase for asymptotic expansion of integrals.

To show that $F$, given by (1), is a distribution in one variable, we assume that $\partial f / \partial x_{1}$ is bounded away from zero in the region under consideration. Recalling our assumption that $(\partial f / \partial x) \neq 0$, we can arrange that $\left(\partial f / \partial x_{1}\right) \neq 0$ by taking a partition of unity in $x, u$ space, and then rotating coordinates in $x$-space.

THeOREM 1.1. If, for $u \in U, a \leqq x_{1} \leqq b$, and for $\left(x_{2}, \cdots, x_{p}\right)$ belonging to an open subset of $R^{p-1}$, we have $\left|\left(\partial f / \partial x_{1}\right)\right| \geqq \alpha>0$, and if $\varphi\left(x_{1}\right) \in C^{\infty}$ with support in $(a, b)$, then $I(\lambda)$, given by the continuation of

$$
I(\lambda)=\gamma(\lambda) \int F(x, \lambda) \varphi\left(x_{1}\right) d x_{1},
$$

depends continuously on $\varphi$ in the $C_{0}^{\infty}$ topology, and smoothly on $x_{2}, \cdots, x_{p} . I(\lambda)$ is an entire analytic function of $\lambda$. We recall that 
$\gamma(\lambda)=1 / \Gamma[(\lambda+1) / 2]$

Proof. We may rewrite (1.1) as a double integral, first choosing $\operatorname{Re}(\lambda)>0$. Then

$$
\begin{aligned}
I(\lambda) & =\gamma(\lambda) \int_{a}^{b} \int_{U}|f(x, u)|^{\lambda} g(x, u) d u \varphi\left(x_{1}\right) d x_{1} \\
& =\gamma(\lambda) \int_{U} \int_{a}^{b}|f(x, u)|^{\lambda} g(x, u) \varphi\left(x_{1}\right) d x_{1} d u .
\end{aligned}
$$

Now we introduce $f$ as a variable of integration;

$$
I(\lambda)=\gamma(\lambda) \int_{U} \int_{\alpha}^{\beta}|f|^{\lambda} \psi\left(f, u, x_{2}, \cdots, x_{p}\right) d f d u,
$$

where

$$
\psi\left(f, u, x_{2}, \cdots, x_{p}\right)=\frac{g(X, u) \varphi\left(x_{1}\right)}{\frac{\partial f}{\partial x_{1}}(X, u)},
$$

$X_{j}=x_{j}(j=2, \cdots, p)$, and $X_{1}\left(f, u, x_{2}, \cdots, x_{p}\right)$ is defined by the relation $f(X, u)=f$. Clearly $\psi$ and its derivatives with respect to $x_{2}, \cdots, x_{p}$ are in $C_{0}^{\infty}$ with respect to $f$, depending continuously on $\varphi$ in the topology of test functions. Hence it suffices to show that an integral of the form

$$
J(\lambda)=\gamma(\lambda) \int|f|^{\lambda} \psi(f) d f
$$

defines an analytic functional of $\psi$. Following I. M. Gelfand and G. E. Shilov [7], we write, with an arbitrary positive integer $k$,

$$
\begin{aligned}
J(\lambda)= & \gamma(\lambda) \int_{-1}^{1}|f|^{\lambda}\left[\psi(f)-\sum_{j=0}^{k} \psi^{(j)}(0) \frac{f^{j}}{j !}\right] d f \\
& +\gamma(\lambda) \sum_{j=0}^{k} \psi^{(j)}(0) \int_{-1}^{1}|f|^{\lambda} \frac{f^{j}}{j !} d f+\gamma(\lambda) \int_{|f| \geqq 1}|f|^{\lambda} \psi(f) d f .
\end{aligned}
$$

The first and third terms are regular in $\lambda$ for $\operatorname{Re}(\lambda)>-k-1$; the second term is easily evaluated as

$$
\gamma(\lambda) \sum_{0 \leqq 2 l \leqq k} \psi^{(2 l)}(0) \frac{1}{(\lambda+2 l+1)(2 l) !} .
$$

Hence, since $\gamma(\lambda)$ has zeros for $\lambda=-2 l-1, l=$ integer $\geqq 0, J(\lambda)$ is an entire functional. Thus $I(\lambda)$ is also an entire functional.

According to the principle of stationary phase, the singularities of $F$ arise from interior points where both $f$ and $\partial f / \partial u$ vanish, or from boundary points where $f$ vanishes and $\partial f / \partial u$ is normal to the boundary. 
(See D. S. Jones and M. Kline [8].) We wish to consider only interior stationary points, and hence we assume that the support of $g(x, u)$ is in the interior of $U$.

THEOREM 1.2. If the support of $g(x, u)$ is in the interior of $U$, and if, at a point $x_{0}, f\left(x_{0}, u\right)$ and $(\partial f / \partial u)\left(x_{0}, u\right)$ do not both vanish anywhere in $U$, then there exists a neighborhood of $x_{0}$ in which $F(x, \lambda)$ is smooth for all $\lambda$.

Proof. Let $K=\inf _{u \in J}\left\{\left|f\left(x_{0}, u\right)\right|^{2}+\left|(\partial f / \partial u)\left(x_{0}, u\right)\right|^{2}\right\}$. At each point $u_{0} \in U$, we have either

(a) $\left|f\left(x_{0}, u_{0}\right)\right|^{2} \geqq K / 2$, or

(b) $\left|(\partial f / \partial u)\left(x_{0}, u_{0}\right)\right| \geqq K / 2$.

Hence we can find a neighborhood of $\left(x_{0}, u_{0}\right)$ in which either

(a) $|f|^{2}>K / 4$, or

(b) $|\partial f / \partial u|^{2}>K / 4$.

Such a neighborhood contains the product of an open ball $B\left(x_{0}\right) \subset R^{p}$, with center at $x_{0}$, and an open ball $B\left(u_{0}\right) \subset R^{n}$, with center at $u_{0}$. The set of such balls $B\left(u_{0}\right)$ forms an open covering of $U$, which can be reduced to a finite covering since $U$ is compact. The intersection of the corresponding $B\left(x_{0}\right)$ is open. We denote this intersection by $C\left(x_{0}\right)$.

Thus, to each $u_{0} \in U$ is associated an open set $N\left(u_{0}\right)$ in which either

(a) $|f|^{2}>K / 4$, or

(b) $|\partial f / \partial u|^{2}>K / 4$, for $x \in C\left(x_{0}\right), u \in N\left(u_{0}\right)$.

We choose a $C^{\infty}$ partition of unity subordinate to our finite covering of $U$. In sets of type (a), the integrand in (1) is $C^{\infty}$ for $x \in C\left(x_{0}\right)$, for all $\lambda$. In sets of type (b), we may introduce $f$ as variable of integration and proceed as in the proof of Theorem 1.1. Here $x$ plays the role of a parameter. Thus integrals over sets of type (b) define functionals which are entire in $\lambda$, and which are $C^{\infty}$ with respect to $x$.

2. Single integrals. In this section, we consider the case $n=1$, i.e. where $U$ is an interval of the real line. We shall obtain a description of the singularity of $F$ near $x_{0}$, associated with a neighborhood of a point $u_{0}$ where $f\left(x_{0}, u_{0}\right)=0$ and $(\partial f / \partial u)\left(x_{0}, u_{0}\right)=0$. According to Theorem 1.2, every singularity of $F$ corresponds to such a neighborhood. First we make a change of variables involving both $x$ and $u$, and obtain an integral of the same type, where $f(x, u)=x_{1}+u^{m}$. Theorem 2.1 states that, for fixed $\lambda, F(x, \lambda)$ is bounded if $g(x, u)$ vanishes sufficiently rapidly at $u=0$. Thus, applying Taylor's theorem to $g$ as function of $u$, we see that the singularities of $F$ arise from terms of the form $\int\left|x_{1}+u^{m}\right|^{\lambda} u^{k} d u$. Finally, Theorems 2.2 and 2.3 show that 
such an integral is the sum of a distribution homogeneous of degree $\lambda+(k+1) / m$ and a regular function.

Without loss of generality, we may assume that $x_{0}=0$ and $u_{0}=0$, and $\left(\partial f / \partial x_{1}\right)(0,0) \neq 0$. We assume further that, at $(0,0)$,

$$
f=\frac{\partial f}{\partial u}=\cdots \frac{\partial^{m-1} f}{\partial u^{m-1}}=0, \quad \frac{1}{m !} \frac{\partial^{m} f}{\partial u^{m}}(0,0) \neq 0 .
$$

We fix $x_{2}=\cdots x_{p}=0$, and denote $x_{1}$ by $x$. From Taylor's theorem,

$$
\begin{aligned}
f(x, u) & =f(0, u)+x e_{1}(x, u) \\
& =e_{1}(x, u)\left(x+\frac{f(0, u)}{e_{1}(x, u)}\right) .
\end{aligned}
$$

Here $e_{1}$ is a smooth function; $e_{1}(0,0)=\left(\partial f / \partial x_{1}\right)(0,0)$. Since $f$ is of order $m$ at the origin, we may write

$$
f(x, u)=e_{1}(x, u)\left(x+u^{m} e_{2}(x, u)\right),
$$

where $e_{2}(x, u)$ is smooth, and $e_{2}(0,0)=\left\{\left[\partial^{m} f(0,0) / \partial u^{m}\right] /\left[m !\left(\partial f / \partial x_{1}\right)(0,0)\right]\right\}$. If $x$ and $u$ are sufficiently small, the implicit function theorem implies that we may introduce a new variable of integration, $v=u\left|e_{2}(x, u)\right|^{1 / m}$; thus we obtain

$$
\gamma(\lambda) \int|f|^{\lambda} g d u=\gamma(\lambda) \int\left|x \pm v^{m}\right|^{\lambda} g_{1}(x, v ; \lambda) d v,
$$

where

$$
g_{1}(x, v ; \lambda)=\left|e_{1}(x, u)\right|^{\lambda} g(x, u) \frac{d u}{d v} .
$$

By replacing $x$ by $-x$ if necessary, we may bring (2.1) into the form where the plus sign holds.

Now we wish to apply Taylor's theorem to $g_{1}(x, v ; \lambda)$, obtaining a polynomial in $v$, with a remainder which vanishes rapidly as $v \rightarrow 0$. First we show that, for fixed $\lambda$, the corresponding term in the expansion of $F$ will be continuous, and can be made as smooth as desired.

THEOREM 2.1. If $g(x, u ; \lambda)$ has $l$ derivatives with respect to $u$, and if $\operatorname{Re}(\lambda)=\lambda_{1}>-l-1$, and if $m \lambda_{1}+k+1>0$, then

$$
I(x, \lambda)=\gamma(\lambda) \int_{0}^{a}\left|x+u^{m}\right|^{\lambda} u^{k} g(x, u, \lambda) d u
$$

is continuous and bounded as a function of $x$.

Proof. We set $\xi=|x|^{1 / m}$, and write $I=I_{1}+I_{2}$, with

$$
I_{1}=\gamma(\lambda) \int_{0}^{2 \hat{\varepsilon}}\left|x+u^{m}\right|^{\lambda} u^{k} g(x, u, \lambda) d u,
$$




$$
I_{2}=\gamma(\lambda) \int_{2 \xi}^{a}\left|x+u^{m}\right|^{\lambda} u^{k} g(x, u, \lambda) d u
$$

In (2.3), we introduce $u=\xi v$. Then

$$
I_{1}=\gamma(\lambda)|x|^{\lambda+(k+1) / m} \int_{0}^{2}\left|\operatorname{sgn} x+v^{m}\right|^{\lambda} v^{k} g(x, \xi v, \lambda) d v .
$$

Continuing this expression with respect to $\lambda$ in the usual way (see proof of Theorem 1.1), we see that if $m \lambda_{1}+k+1>0, I_{1}$ is continuous and bounded. We may rewrite (2.4) as

$$
I_{2}=\gamma(\lambda) \int_{2 \xi}^{a}\left|1+\frac{x}{u^{m}}\right|^{\lambda} u^{k+m \lambda} g(x, u ; \lambda) d u .
$$

Hence,

$$
\left|I_{2}\right| \leqq \gamma(\lambda)\left|1-\frac{1}{2^{m}}\right|_{0 \leqq u \leqq a}^{\lambda_{1}} \sup _{0}|g(x, u ; \lambda)| \int_{0}^{a} u^{k+m \lambda_{1}} d u
$$

which is clearly bounded if $k+m \lambda_{1}+1>0$. The continuity of $I_{2}$ follows similarly from the uniform continuity of the integrand.

We remark that smoothness of (2.2) for sufficiently large $k$ follows from formal differentiation of (2.2), and application of Theorem 2.1.

Applying Taylor's theorem to $g_{1}(x, v ; \lambda)$ appearing in $(2.1)$, we see that

$$
\begin{aligned}
\gamma(\lambda) \int \mid f^{\lambda} g(x, u, \lambda) d u= & \sum_{j=0}^{k} g^{(j)}(x, \lambda) \gamma(\lambda) \int\left|x+v^{m}\right|^{\lambda} v^{j} d v \\
& +\gamma(\lambda) \int\left|x+v^{m}\right|^{\lambda} v^{k+1} g_{2}(x, v, \lambda) d v
\end{aligned}
$$

Theorem 2.1 implies that the remainder is smooth in $x$ for fixed $\lambda$, if $k$ is sufficiently large. Evaluation of the singularities of $F$ is therefore reduced to evaluation of the singularities of integrals of the form

$$
I(x, \lambda)=m \gamma(\lambda) \int_{0}^{a}\left|x+v^{m}\right| \lambda v^{n-1} d v .
$$

A change of variables yields an integral of the form

$$
I(x, \lambda)=\gamma(\lambda) \int_{0}^{a}|x+u|^{\lambda} u^{\alpha-1} d u,
$$

where $\alpha=(n / m)$.

In order to describe the singularities of (2.7) and related integrals, we shall require some facts about certain homogeneous distributions. We set

$$
x_{+}=\max (x, 0), \quad x_{-}=\max (-x, 0) .
$$


Lemma 2.1. The functionals $[1 / \Gamma(\lambda+1)] x_{+}^{\lambda}$ and $[1 / \Gamma(\lambda+1)] x_{-}^{\lambda}$ are entire analytic functionals. Moreover,

$$
\left\{\begin{array}{l}
\left.\frac{1}{\Gamma(\lambda+1)} x_{+}^{\lambda}\right|_{\lambda=-p}=\delta^{(p-1)}(x) \quad(p=1,2, \cdots) \\
\left.\frac{1}{\Gamma(\lambda+1)} x_{-}^{\lambda}\right|_{\lambda=-p}=(-1)^{p-1} \delta^{(p-1)}(x) \quad(p=1,2, \cdots) .
\end{array}\right.
$$

The proof is in I. M. Gelfand and G. E. Shilov [7], pp. 56-65. It is similar to the latter part of the proof of Theorem 1.1.

The following theorem leads immediately to results about (2.7).

THEOREM 2.2. If $\operatorname{Re}(\alpha)>0$, the integral

$$
J_{+}(x, \lambda)=\int_{0}^{a} \frac{(x+u)_{+}^{\lambda}}{\Gamma(\lambda+1)} \frac{u^{\alpha-1}}{\Gamma(\alpha)} d u
$$

may be represented in the form

$$
\begin{aligned}
J_{+}(x, \lambda)= & a_{+}(\lambda, \alpha) \frac{x_{+}^{\lambda+\infty}}{\Gamma(\lambda+\alpha+1)} \\
& +a_{-}(\lambda, \alpha) \frac{x_{-}^{\lambda+\alpha}}{\Gamma(\lambda+\alpha+1)}+R(x, \lambda, \alpha) .
\end{aligned}
$$

Here $R(x, \lambda, \alpha)$ is a smooth function of $x$ for small $x$, which is regular in $\lambda$ and $\alpha$, except for simple poles where $\lambda+\alpha$ is a nonnegative integer. The coefficients $\alpha_{+}$and $a_{-}$are regular except for simple poles where $\lambda+\alpha$ is an integer. The sum of the residues at the poles is zero, since $J_{+}(x, \lambda)$ is regular. We have

$$
a_{+}(\lambda, \alpha)=\frac{\sin \pi \lambda}{\sin \pi(\lambda+\alpha)}, \quad a_{-}(\lambda, \alpha)=\frac{-\sin \pi \alpha}{\sin \pi(\lambda+\alpha)} .
$$

We also have, for small $x$,

$$
J_{-}(x, \lambda)=\int_{0}^{a} \frac{(x+u)_{-}^{\lambda}}{\Gamma(\lambda+1)} \frac{u^{\alpha-1}}{\Gamma(\alpha)} d u=\frac{x_{-}^{\lambda+\alpha}}{\Gamma(\lambda+\alpha+1)} .
$$

Proof. We shall use analytic continuation in $\lambda$ and $\alpha$. First we assume that $-1<\operatorname{Re}(\lambda)<-1 / 2, \quad 0<\operatorname{Re}(\alpha)<1 / 2$. Then we may write

$$
J_{+}(x, \lambda)=\int_{0}^{\infty} \frac{(x+u)_{+}^{\lambda}}{\Gamma(\lambda+1)} \frac{u^{\alpha-1}}{\Gamma(\alpha)} d u+R(x, \lambda, \alpha),
$$

with 


$$
R(x, \lambda, \alpha)=-\int_{a}^{\infty} \frac{(x+u)_{+}^{\lambda}}{\Gamma(\lambda+1)} \frac{u^{\alpha-1}}{\Gamma(\alpha)} d u
$$

The first integral in (2.13) may be treated by setting $u=|x| v$. The resulting coefficient of $|x|^{\lambda+\infty}$ may be evaluated in terms of $\Gamma$-functions, to produce (2.11). To see that $R(x, \lambda, \alpha)$ is smooth in $x$, we introduce $v=(1 / u)$ as variable of integration in $(2.14)$; thus

$$
R(x, \lambda, \alpha)=-\int_{0}^{1 / \alpha} \frac{(1+v x)_{+}^{\lambda}}{\Gamma(\lambda+1)} \frac{v^{-(\alpha+\lambda+1)}}{\Gamma(\alpha)} d v
$$

We may apply Taylor's theorem to $(1+v x)_{+}^{\lambda}$, obtaining a polynomial in $v x$, plus a remainder which vanishes rapidly for $v=0$. Hence, the residues of $R$ at its poles are powers of $x$, and the remainder is smooth in $x$.

Now we continue our representation (2.13) for $\operatorname{Re}(\alpha)>0$. Equation (2.9) shows that $J_{+}(x, \lambda)$ is regular for $-1<\lambda<-1 / 2$ and $\operatorname{Re}(\alpha)>0$. On the other hand, the coefficients $a_{ \pm}(x, \alpha)$ have simple poles for $\lambda+\alpha=$ integer. The residues at these poles are determined by the behavior at $\infty$ of the integrand in (2.13). Comparing (2.13) and (2.14), we see that the sum of the residues at the poles is zero.

Now we are ready to continue in $\lambda$, for fixed $\alpha$, with $\operatorname{Re}(\alpha)>0$. First we assume that $\alpha$ is not an integer. From (2.10) and (2.11), it is apparent that the only possible singularities of the representation (2.10) are where $\lambda+\alpha$ is an integer. The case where $\lambda+\alpha$ is a nonnegative integer has already been discussed. If $\lambda+\alpha$ is a negative integer, then both $J(x, \lambda)$ and $R(x, \lambda, \alpha)$ are regular. It follows that the sum of the residues of

$$
a_{+} \frac{x_{+}^{\lambda+\alpha}}{\Gamma(x+\alpha+1)} \quad \text { and } \quad a_{-} \frac{x_{-}^{\lambda+\alpha}}{\Gamma(\lambda+\alpha+1)}
$$

must be zero. This can be verified by a direct calculation, using Lemma 2.1.

If $\alpha$ is a positive integer, $\alpha=l$, we obtain

$$
a_{+}(x, l)=(-1)^{l}, \quad a_{-}(\lambda, l)=0 .
$$

In this case, $R$ is regular in $\lambda$, because of the factor $1 / \Gamma(\lambda+1)$.

The fact that $(x+u)_{+}^{\lambda}+(x+u)_{-}^{\lambda}=|x+u|^{\lambda}$ immediately implies

\section{THEOREM 2.3. If}

$$
I(x, \lambda)=\gamma(\lambda) \int_{0}^{a}|x+u|^{\lambda} u^{\alpha-1} d u
$$




$$
\begin{aligned}
I(x, \lambda)= & b_{+}(\lambda, \alpha) \frac{x_{-}^{\lambda+\alpha}}{\Gamma(\lambda+\alpha+1)} \\
& +b_{-}(\lambda, \alpha) \frac{x_{-}^{\lambda+\alpha}}{\Gamma(\lambda+\alpha+1)}+R(x, \lambda, \alpha) .
\end{aligned}
$$

Here

$$
\begin{gathered}
b_{+}(\lambda, \alpha)=\Gamma(\alpha) \gamma(\lambda) \Gamma(\lambda+1) \frac{\sin \lambda \pi}{\sin \pi(\lambda+\alpha)} \\
b_{-}(\lambda, \alpha)=\Gamma(\alpha) \gamma(\lambda) \Gamma(\lambda+1)\left[1-\frac{\sin \pi \alpha}{\sin \pi(\lambda+\alpha)}\right],
\end{gathered}
$$

and $R(x, \lambda, \alpha)$ is a smooth function of $x$, with poles if $\lambda+\alpha$ is $a$ nonnegative integer.

REMARK. Equation (2.16) may be differentiated with respect to $\alpha$, to obtain results for

$$
\gamma(\lambda) \int_{0}^{a}|x+u|^{\lambda} u^{\alpha-1} \log u d u
$$

We omit the calculation.

It may be useful to give our results for the leading, or most singular term in the expansion of (1) an explicit form. In this term, only the values of $\left(\partial f / \partial x_{1}\right)(0,0)=b,(1 / m !)\left(\partial^{m} f / \partial u^{m}\right)(0,0)=c$, and $g(0,0)$ enter. Taking the most singular term only,

$$
F_{1}\left(x_{1}\right) \sim \gamma(\lambda) \int_{-a}^{a}| \pm b x+| c\left|u^{m}\right|^{\lambda} d u g(0,0) .
$$

Setting $v=|c|^{1 / m} u$, and $z=b \operatorname{sgn}(c) x$,

$$
F_{1}\left(x_{1}\right) \sim \gamma(\lambda) \int_{-a_{1}}^{a_{1}}\left|z+v^{m}\right|^{\lambda} d v \frac{g(0,0)}{|c| \frac{1}{m}} .
$$

If $m$ is even,

$$
F_{1}\left(x_{1}\right) \sim 2 \gamma(\lambda) \int_{0}^{a_{1}}\left|z+v^{m}\right|^{\lambda} d v \frac{g(0,0)}{|c| \frac{1}{m}},
$$

and if $m$ is odd,

$$
F_{1}\left(x_{1}\right) \sim \gamma(\lambda) \int_{0}^{a}\left(\left|z+v^{m}\right|^{\lambda}+\left|-z+v^{m}\right|^{\lambda}\right) d v \frac{g(0,0)}{|c| \frac{1}{m}} .
$$

These integrals may be evaluated by means of Theorem 2.3. 
3. Reduction of double integrals to a standard form. We shall consider the integral (1), in the case $n=2$. As before, the singularity of $F$ near a given point $x_{0}$ is associated with points $u_{0}$ such that $f\left(x_{0}, u_{0}\right)=0$ and $(\partial f / \partial u)\left(x_{0}, u_{0}\right)=0$. Such points $u_{0}$ may be isolated, or may lie on a curve. In order to evaluate the contribution from a neighborhood of such a curve, we would have to cover it by a system of sufficiently small neighborhoods, taking particular notice of singular points of the curve, and then apply the theory of this section.

Without loss of generality, we may assume that $x_{0}=0$ and $u_{0}=0$. We set $f_{0}(u)=f(0, u)$. Our method consists in dividing the $u$-plane into regions, in such a way that distinct roots of $f_{0}$ appear in different regions. After a change of variables, $f_{0}$ may be represented as the product of a monomial and a nonvanishing function, in each region. The shapes of the regions involved are determined by the Puiseux expansions of the roots of $f_{0}$. We obtained the required regions by an iterative process. If $f_{0}$ is analytic, then the process will terminate. In fact, if distinct roots of $f_{0}$ have distinct Puiseux expansions, then the process will terminate if $f_{0} \in C^{\infty}$. Since the process involves only a finite number of derivatives of $f_{0}$, it will terminate if $f_{0}$ has enough derivatives so that distinct roots have distinct truncated Puiseux expansions.

The integral over a single region assumes the form

$$
\gamma(\lambda) \iint\left|x+u^{\alpha} v^{\beta}\right|^{\lambda} u^{\gamma-1} v^{\delta-1} g(u, v ; \lambda, x) d u d v .
$$

Integrals of this form will be treated in $\S 4$. Finally, (Lemma 3.1) we show that if $f_{0}$ has order $m$ at the origin, then $\min (\gamma / \alpha, \delta / \beta) \geqq 1 / m$.

As before, we assume that $\left(\partial f / \partial x_{1}\right)(0,0) \neq 0$, we set $x_{2}=x_{3}=$ $\cdots x_{p}=0$, and we write $x_{1}=x$. Then we may write

$$
f(x, u)=f_{0}(u)+x e_{1}(x, u)=e_{1}(x, u)\left(x+f_{0}(u) E(x, u)\right) .
$$

Functions denoted by $e_{i}$ or $E_{i}$ are different from zero at the origin. We first consider the simplest case, where the roots of $f_{0}$ have distinct tangents at the origin. We write $u_{1}=u, u_{2}=v$. Then $f_{0}\left(u_{1}, u_{2}\right)=$ $P_{m}(u, v)+Q(u, v)$, where $P_{m}$ is a homogeneous polynomial of degree $m$, and $Q$ is of order $m+1$ at the origin. By our assumption, the real roots of $P_{m}$ are distinct. We introduce a partition of unity on the circle, symmetric about the origin, such that each function of the partition has its support in a region where either $P_{m}(\cos \theta, \sin \theta) \neq 0$, or $(\partial / \partial \theta)\left(P_{m}(\cos \theta, \sin \theta)\right) \neq 0$. Regions of the first type give rise to an integral of the form

$$
\gamma(\lambda) \iint\left|x+r^{m} E_{1}(x, r, \theta)\right|^{\lambda}\left|e_{1}\right|^{\lambda} g_{1} r d \theta d r .
$$


In regions of the second type, we may introduce $V=P_{m}(\theta)+r Q(r, \theta)$ as a variable of integration; we obtain an integral of the form

$$
\gamma(\lambda) \iint\left|x+r^{m} V E_{1}\right|^{\lambda} g_{1}(x, r, V, \lambda) r d V d r,
$$

if $r$ is sufficiently small in the support of $g_{1}$.

Now we consider the general case, where $P_{m}$ may have multiple roots. We shall obtain integrals similar to (3.2) and (3.3), which may be reduced to the form (3.1). By the term "sector" we shall mean a region generated by rotating a line about the origin. Thus a sector will consist of two wedge-shaped regions. By a "strip" we shall mean a region generated by displacement of a line parallel to the $u$-axis. By a "quadratic transformation" we shall mean a transformation of the form $u=u_{1}, v=u_{1} v_{1}$. Under a quadratic transformation, a sector in the $u, v$ plane which does not contain the $v$-axis is transformed into a strip in the $u_{1}, v_{1}$ plane. We shall be integrating over strips and sectors, and we would like to decompose an integral over a strip into a sum of integrals over sectors. We accomplish this by formally extending all integrations over the whole plane. First, we assume that the integrand in (1) has support in a finite disc about the origin. Given any open, finite covering of the unit circle, we can find a $C^{\infty}$ partition of unity, such that each function $\varphi_{j}(\theta)$ has its support in one of the covering sets. The functions $\varphi_{j}(2 \theta)$ provide a partition of unity which is constant on lines through the origin, and such that each function of the partition has its support in a sector. After rotation and application of a quadratic transformation, each of the functions $\varphi_{j}$ will have support in a strip. Thus, after quadratic transformation, our original integral is transformed into a sum of integrals over strips. Integration over each strip may formally be extended over the whole plane, which in turn may be decomposed into sectors by a partition of unity. This process may be repeated as often as desired. In this way the burden of the complexities of the actual region of integration is thrown on the structure of the final partition of unity.

We cover each of the real roots of $P_{m}(u, v)$ by a sufficiently small open sector, and choose a covering of the remaining sectors which is finite and does not intersect the roots of $P_{m}$. We choose a partition of unity subordinate to this covering. Integrals over sectors which do not contain a root of $P_{m}$, or which contain a simple root of $P_{m}$, may be treated as before, leading to integrals of the form (3.2) or (3.3). A sector which contains a multiple root of $P_{m}$ may be rotated so that the root coincides with the new $u$-axis. Under such a transformation, an expression of the form $u^{\alpha} v^{\beta} E(u, v)$, where $E(0,0) \neq 0$, is transformed into a similar expression. Such expressions remain of the same type under a quadratic expression as well. Hence, after a rotation 
and a quadratic transformation, we have

$$
E(u, v, x) f_{0}(u, v)=E\left(P_{m}+Q\right)=u_{1}^{m} E_{1}\left[P_{m_{1}}\left(u_{1}, v_{1}\right)+Q_{1}\right] .
$$

Here we have divided $P_{m}+Q$ by $u_{1}^{m}$ and collected terms of lowest degree in $u_{1}$ and $v_{1}$ to obtain $P_{m_{1}}\left(u_{1}, v_{1}\right)$. Observe that $m_{1}$ is less than or equal to the multiplicity of the root of $P_{m}$ in question.

Now we apply a similar procedure to $P_{m_{1}}$ instead of $P_{m}$. A second application of the procedure may result in an expression of the form

$$
E f_{0}=u_{2}^{\alpha} v_{2}^{\beta} E_{2}\left[P_{m_{2}}+Q\right],
$$

if $v_{1}$ divides $P_{m_{1}}$, but further applications of the procedure do not result in expressions of more complicated form. We temporarily halt our procedure if $E f_{0}$ assumes the form

$$
E f_{0}=u_{l}^{\alpha} v_{l}^{\beta} E_{l}\left(v_{l}+a u_{l}+\cdots\right)^{\gamma},
$$

where $a \neq 0$. This situation will always occur if distinct roots of $f_{0}$ have distinct (truncated) Puiseux expansions. In particular, if $f_{0}$ is analytic, we may apply the Weierstrass preparation theorem to $f_{0}$ (see G. A. Bliss [1], pp. 53-55.) Thus after rotation,

$$
f_{0}(u, v)=E(u, v)\left[u^{m}+a_{1}(v) u^{m-1}+\cdots+a_{m}(v)\right],
$$

where $E(u, v)$ and $a_{j}(v)(j=1, \cdots, m)$ are analytic and $E(0,0) \neq 0$. Since the field of fractional power series is algebraically closed, the roots of $f_{0}$ may be expanded in Puiseux series (see R. Walker [12], pp. 97-102.) Thus after a finite number of quadratic transformations, the distinct roots of $f_{0}$ must belong to distinct sectors, and $f_{0}$ will appear as a product of powers of factors whose lowest term is of degree one, multiplied by a nonvanishing function, as shown in (3.4). Hence, after a final rotation and quadratic transformation, we are led to integrals of the form

$$
\gamma(\lambda) \int\left|x+u^{\alpha} v^{\beta} E(u, v, x)\right|^{\lambda} u^{\gamma-1} v^{\delta-1} g(u, v, x, \lambda) d u d v,
$$

where $\gamma \geqq 1, \delta \geqq 1$. The factor $u^{\gamma-1} v^{\varsigma-1}$ arises from the Jacobians of the quadratic transformations. Now, using the implicit function theorem, we set $v_{1}=v|E(u, v, x)|^{1 / \beta}$, for $u, v, x$ sufficiently small and obtain an integral of the form

$$
\gamma(\lambda) \iint\left| \pm x+u^{\alpha} v_{1}^{\beta}\right|^{\lambda} u^{\gamma-1} v_{1}^{\delta-1} g\left(u, v_{1}, x, \lambda\right) d u d v_{1} .
$$

Thus after appropriate changes of variables, and a partition of unity, the evaluation of (1) is reduced to evaluation of integrals of the form (3.5). 
We shall see in the next section that the leading singularity of (3.5) is determined by $\mu=\min (\gamma / \alpha, \delta / \beta)$.

LEMMA 3.1. If $f_{0}$ has order $m$ at the origin, and $g(u, v, s, \lambda)$ has the form $u^{\gamma-1} v^{s-1} g_{1}(u, v, x, \lambda)$, then in all integrals of the form (3.5) which arise by the preceding process, we have $\mu \geqq \min (\gamma / m, \delta / m)$.

Proof. All integrals which arise are of the form

$$
I_{n}=\gamma(\lambda) \iint\left|x+u^{\alpha} v^{\beta} E\left(P_{l}+Q\right)\right|^{\lambda} u^{\gamma-1} v^{\delta-1} g(u, v, x, \lambda) d u d v .
$$

For such an integral, we define $\mu_{n}=\min [\gamma /(\alpha+l), \delta /(\beta+l)]$. We show that $\mu$ is a nondecreasing function under rotations, quadratic transformations, and (clearly) if a monomial is factored out of $P_{l}+Q$. The only nontrivial case is a quadratic transformation. Under quadratic transformation,

$$
I_{n+1}=\gamma(\lambda) \iint\left|x+u_{1}^{\alpha+\beta+l} v_{1}^{\beta} E\left(P_{l_{1}}+Q_{1}\right)\right|^{\lambda} u_{1}^{\gamma+\delta-1} v_{1}^{\delta-1} g\left(u_{1}, u_{1} v_{1}, x, \lambda\right) d u_{1} d v_{1} .
$$

Hence, $\quad \mu_{n+1}=\min \left[(\gamma+\delta) /\left(\alpha+\beta+l+l_{1}\right), \delta /\left(\beta+l_{1}\right)\right]$. Since $l_{1} \leqq l$, we have $\delta /\left(\beta+l_{1}\right) \geqq \mu_{n}$, hence also $(\gamma+\delta) /\left(\alpha+\beta+l+l_{1}\right) \geqq \mu_{n}$.

4. Expansion of double integrals. In this section, we shall. expand double integrals of the form

$$
I(x)=\gamma(\lambda) \iint\left|x+u^{\alpha} v^{\beta}\right|^{\lambda} u^{\gamma-1} v^{\delta-1} g(u, v ; \lambda, x) d u d v,
$$

in powers of $x$, using the results of $\S 2$. First we prove Theorem 4.1, which asserts that $I(x)$ is continuous in $x$ if $\gamma+\alpha \operatorname{Re}(\lambda)>0$ and $\delta+\beta R e(\lambda)>0$. Thus if $g(u, v ; \lambda, x)$ is written as a sum of functions, with remainder multiplied by a large power of both $u$ and $v$, then the remainder will give rise to a continuous function of $x$. The major portion of this section is devoted to expansion of integrals of the form

$$
J(x)=\gamma(\lambda) \iint_{\substack{u \geq 0 \\ v \geqq 0}}\left|x+u^{\alpha} v^{\beta}\right|^{\lambda} u^{\gamma-1} v^{\delta-1} g(v ; \lambda, x) \varphi(u) d u d v,
$$

where $g(v)$ and $\varphi(u)$ have compact support, and $\varphi(\mu) \equiv 1$ for small $u$. The results are summarized as Lemma 4.2. An appropriate expansion of $g(u, v ; \lambda, x)$, together with Lemma 4.2 then implies an expansion of (4.1) in powers of $x$, specified in Theorem 4.2. Finally, we give a more or less explicit formula for the coefficient of the leading or most singular term in the expansion of (4.1).

THEOREM 4.1. If $\alpha, \beta \geqq 0, \gamma, \delta>0$ and if $\gamma+\alpha \operatorname{Re}(\lambda)>0$ and 
$\delta+\beta \operatorname{Re}(\lambda)>0$, and if $g(u, v ; \lambda)$ has enough derivatives with respect to $u$ and $v$, and has compact support in $u$ and $v$, then the integral (4.1) is bounded and continuous in $x$, for small $x$.

Proof. Let $\xi=|x|^{1 / \alpha}$. We write

$$
\begin{aligned}
I(x)= & \gamma(\lambda) \iint_{|u| \leqq k \xi}\left|x+u^{\alpha} v^{\beta}\right|^{\lambda} u^{\gamma-1} v^{\delta-1} g d u d v \\
& +\gamma(\lambda) \iint_{u>k \xi}\left|x+u^{\alpha} v^{\beta}\right|^{\lambda} u^{\gamma-1} v^{\delta-1} g d u d v, \\
I(x)= & I_{1}+I_{2} .
\end{aligned}
$$

We shall specify $k$ presently. In the first integral, we set $u=\xi \mu$. Then

$$
I_{1}=\gamma(\lambda) \iint_{|\mu| \leqq k} \xi^{\alpha \lambda+\gamma}\left| \pm 1+\mu^{\alpha} v^{\beta}\right|^{\lambda} \mu^{\gamma-1} v^{\varsigma-1} g d \mu d v .
$$

Now we choose $k$ so small that $\pm 1+\mu^{\alpha} v^{\beta}$ does not vanish for $|\mu| \leqq k$, if $v$ is in the support of $g$. As in the proof of Theorem 2.1, it follows that $I_{1}$ is continuous in $x$.

In the second integral, we divide by $|u|^{\alpha}$; thus

$$
I_{2}=\gamma(\lambda) \iint_{|u|>k \xi}\left| \pm \frac{x}{|u|^{\alpha}}+v^{\beta}\right|^{\lambda} v^{\wedge-1} g d v( \pm 1)|u|^{\alpha \lambda+\gamma-1} d u \text {. }
$$

Now we may apply Theorem 2.1 to the inner integral taken over $v$, since $x|u|^{-\alpha}$ is bounded. Since $\beta \operatorname{Re}(\lambda)+\delta>0$, the inner integral is continuous in $x$ and $u$ for $u$ bounded away from zero, and bounded for $u$ in the region of integration. Hence, since $\alpha R e(\lambda)+\gamma>0$, the double integral is continuous in $x$.

We proceed to the statement and proof of Lemma 4.1. Starting with (4.2), we set $\mu=u^{\alpha}, \nu=v^{\beta}, p=\gamma / \alpha, q=\delta / \beta, r=1 / \beta, \varphi_{1}(\mu)=$ $\varphi\left(\mu^{1 / \alpha}\right)$. Thus

$$
J(x)=\gamma(\lambda) \iint_{\substack{\mu \geqq 0 \\ \nu \geqq 0}}|x+\mu \nu|^{\lambda} \mu^{p-1} \nu^{q-1} g\left(\nu^{r} ; x, \lambda\right) \varphi_{1}(\mu) \frac{d \mu d \nu}{\alpha \beta} .
$$

We recall that $\phi_{1}(\mu) \equiv 1$ for small $\mu$. Introducing $w=\mu \nu$ as a new variable of integration, we have

$$
J(x)=\gamma(\lambda) \int_{0}^{\infty}|x+w|^{\lambda} k(w ; x, \lambda) d w,
$$

where

$$
k(w ; x, \lambda)=\int_{0}^{\infty} g\left(\frac{w^{r}}{\mu^{r}} ; x, \lambda\right) \rho_{1}(\mu) w^{q-1} \mu^{p-q-1} \frac{d \mu}{\alpha \beta} .
$$

For $w \neq 0$, the integral exists and is smooth, since $g$ and $\varphi_{1}$ have 
compact support. For the same reason, $k$ has compact support in $w$. It follows that the singularities of $J(x)$ for small $x$ are determined by the behavior of $k(w ; x, \lambda)$ for small $w$. This is precisely the statement that the singularities of (4.2) are associated with the $u$ and $v$ axes. Since $x$ and $\lambda$ play the role of parameters in the following, we shall usually not indicate their presence.

Lemma 4.1. For small $w$, the function $k(w)$, defined by (4.4), with $p, q, r>0$, may be represented in the form

$$
\begin{aligned}
k(w)= & a_{\supset} w^{p-1}+a_{0}^{1} w^{p-1} \log w \\
& +\sum_{l=0}^{L} b_{l} w^{q+l r-1}+w^{q+(L+1) r-1} R\left(w^{r}\right),
\end{aligned}
$$

for any $L$, if $g$ has enough derivatives. The coefficients $a_{0}, a_{0}^{1}, b_{l}$ depend on $x$ and $\lambda$, and are given by certain of the formulas (4.64.23). The coefficient $a_{0}^{1}$ vanishes unless $p=q+J r$, for some integer $J \geqq 0$. The remainder $R\left(w^{r}\right)$ is smooth for small values of its argument.

Proof. We distinguish three cases:

(A) $q>p$,

(B) $q<p, p \neq q+J r$ for any integer $J$, and

(C) $q=p+J r, J=$ integer $\geqq 0$.

A. If $q>p$, we define

$$
k_{0}(w)=w^{q-1} \int_{0}^{\infty} g\left(\frac{w^{r}}{\mu^{r}}\right) \mu^{p-q-1} \frac{d \mu}{\alpha \beta} .
$$

If $w \neq 0$, this integral exists, since $g$ has compact support. Making a change of variables,

$$
k_{0}(w)=w^{p-1} \int_{0}^{\infty} g\left(\nu^{r}\right) \nu^{q-p-1} \frac{d \nu}{\alpha \beta} .
$$

Hence, we have $k_{0}(w)=w^{p-1} a_{0}(x, \lambda)$, with

$$
a_{0}(x, \lambda)=\int_{0}^{\infty} g\left(\nu^{r} ; x, \lambda\right) \nu^{q-p-1} \frac{d \nu}{\alpha \beta} .
$$

Now we may write

$$
k(w)=a_{\mathfrak{\varepsilon}} w^{p-1}+w^{q-1} \int_{0}^{\infty} g\left(\frac{w^{r}}{\mu^{r}}\right)\left[\varphi_{1}(\mu)-1\right] \mu^{p-q-1} \frac{d \mu}{\alpha \beta} .
$$

We observe that $\varphi_{1}(\mu)-1$ vanishes for small $\mu$. Hence, $g$ may be expanded in powers of $w^{r} / \mu^{r}$, leading to an expansion of $k(w)$ in powers of $w^{r}$. We have 


$$
b_{l}(x, \lambda)=\left.\frac{1}{l !}\left(\frac{\partial}{\partial v}\right)^{l} g(v ; x, \lambda)\right|_{v=0} \int_{0}^{\infty}\left[\varphi_{1}(\mu)-1\right] \mu^{p-q-l r-1} \frac{d \mu}{\alpha \beta} .
$$

B. If $q<p, q+(J-1) r<p<q+J r$, for some positive integer $J$, we write

$$
\begin{aligned}
g(v) \varphi(\mu)=\left(\sum_{0}^{J-1} g_{l} v^{l}\right) \varphi(u) & +\left(g(v)-\sum_{0}^{J-1} g_{l} v^{l}\right) \\
& +\left(g(v)-\sum_{0}^{J-1} g_{l} v^{l}\right)(\varphi(u)-1) ;
\end{aligned}
$$

here

$$
g_{l}(x, \lambda)=\left.\frac{1}{l !}\left(\frac{\partial}{\partial v}\right)^{l} g(v ; x, \lambda)\right|_{v=0} .
$$

Thus $k(w)=k_{1}(w)+k_{2}(w)+k_{3}(w)$, with

$$
\begin{aligned}
& k_{1}(w)=\int_{0}^{\infty} \sum_{l=0}^{J-1} g_{l} \frac{w^{l r}}{\mu^{l r}} \varphi_{1}(\mu) w^{q-1} \mu^{p-q-1} \frac{d \mu}{\alpha \beta}, \\
& k_{2}(w)=\int_{0}^{\infty}\left[g\left(\frac{w^{r}}{\mu^{r}}\right)-\Sigma g_{l} \frac{w^{l r}}{\mu^{l r}}\right] w^{q-1} \mu^{p-q-1} \frac{d \mu}{\alpha \beta}, \\
& k_{3}(w)=\int_{0}^{\infty}\left[g\left(\frac{w^{r}}{\mu^{r}}\right)-\Sigma g_{l} \frac{w^{l r}}{\mu^{l r}}\right]\left[\varphi_{1}(\mu)-1\right] \mu^{p-q-1} \frac{d \mu}{\alpha \beta} .
\end{aligned}
$$

The integral (4.9) exists, since for $0 \leqq l \leqq J-1, p-l r-q>0$, and $\varphi_{1}$ has compact support. In fact, we have

$$
k_{1}(w)=\sum_{l=0}^{J-1} b_{l} w^{q+l r-1},
$$

with

$$
b_{l}(x, \lambda)=g_{l}(x, \lambda) \int_{0}^{\infty} \varphi_{1}(\mu) \mu^{p-l r-q-1} \frac{d \mu}{\alpha \beta}, \quad(0 \leqq l \leqq J-1) .
$$

The integral (4.10) exists for $w \neq 0$, since, for small $\mu, g\left(w^{r} / \mu^{r}\right)$ vanishes, and $p-l r-q>0$. For large $\mu$, the quantity inside the brackets may be written as $w^{J} \mu^{-J} h(w / \mu)$, where $h$ is a smooth function. Hence, $k_{2}$ is integrable at $\infty$. A change of variables shows that

$$
k_{2}(w)=w^{p-1} \int_{0}^{\infty}\left[g\left(\nu^{r} ; x, \lambda\right)-\Sigma g_{l}(x, \lambda) \nu^{l r}\right] \nu^{q-p-1} \frac{d \nu}{\alpha \beta} ;
$$

thus

$$
a_{0}(x, \lambda)=\int_{0}^{\infty}\left[g\left(\nu^{r} ; x, \lambda\right)-\Sigma g_{l}(x, \lambda) \nu^{l r}\right] \nu^{q-p-1} \frac{d \nu}{\alpha \beta}
$$


Finally, we observe that (4.11) may be written in the form

$$
k_{3}(w)=w^{q+J r-1} \int_{0}^{\infty} h\left(\frac{w^{r}}{\mu^{r}}\right)\left[\varphi_{1}(\mu)-1\right] \mu^{p-J r-q-1} \frac{d \mu}{\alpha \beta} .
$$

This integral may be treated in the same manner as (4.7).

C. If $p=q+J r, J$ is a nonnegative integer. This case is similar to the preceding one. We shall use the Heaviside function

$$
H(1-v)= \begin{cases}0 & \text { if } v \geqq 1 \\ 1 & \text { if } v<1\end{cases}
$$

We may write

$$
\begin{aligned}
\varphi(u) g(v)= & \sum_{l=0}^{J-1}\left(g_{l} v^{l}\right) \varphi(u)+\left[g(v)-\sum_{l=0}^{J-1} g_{l} v^{l}-H(1-v) g_{J} v^{J}\right] \\
& +\left[g(v)-\sum_{l=0}^{J-1} g_{l} v^{l}-H(1-v) g_{J} v^{J}\right][\varphi(u)-1] \\
& +H(1-v) g_{J} v^{J} H(1-u)+H(1-v) g_{J} v^{J}[\varphi(u)-H(1-u)] .
\end{aligned}
$$

Thus $k(w)=\sum_{j=1}^{5} k_{j}(w)$, with

$$
\begin{aligned}
k_{1}(w)= & \int_{0}^{\infty}\left(\sum_{l} g_{l} w^{l r} \mu^{-l r}\right) \varphi_{1}(\mu) w^{q-1} \mu^{p-q-1} \frac{d \mu}{\alpha \beta}, \\
k_{2}(w)= & \int_{0}^{\infty}\left[g\left(w^{r} \mu^{-r}\right)-\Sigma g_{l} w^{l r} \mu^{-l r}\right. \\
& \left.-H\left(1-w \mu^{-1}\right) g_{J} w^{J r} \mu^{-J r}\right] w^{q-1} \mu^{p-q-1} \frac{d \mu}{\alpha \beta}, \\
k_{3}(w)= & \int_{0}^{\infty}\left[g\left(w^{r} \mu^{-r}\right)-\Sigma g_{l} w^{l r} \mu^{-l r}\right. \\
& \left.-H\left(1-w \mu^{-1}\right) g_{J} w^{J r} \mu^{-J r}\right]\left(\varphi_{1}(\mu)-1\right) w^{q-1} \mu^{p-q-1} \frac{d \mu}{\alpha \beta},
\end{aligned}
$$

$$
k_{4}(w)=\int_{0}^{\infty} H\left(1-\frac{w}{\mu}\right) g_{J} w^{J r} \mu^{-J r} H(1-\mu) w^{q-1} \mu^{p-q-1} \frac{d \mu}{\alpha \beta},
$$

$$
\begin{aligned}
k_{5}(w)= & \int_{0}^{\infty} H\left(1-w \mu^{-1}\right) g_{J} w^{J r} \mu^{-J r} \\
& \times\left[\varphi_{1}(\mu)-H(1-\mu)\right] w^{q-1} \mu^{p-q-1} \frac{d \mu}{\alpha \beta} .
\end{aligned}
$$

The integral (4.15) is identical with (4.9); thus the coefficients $b_{l}(l=0, \cdots, J-1)$ are given by (4.12). The integral (4.16) is similar to (4.10). By analogous reasoning, we conclude that $k_{2}(w)=w^{p-1} c_{0}(x, \lambda)$, with

(4.20) $\quad c_{0}(x, \lambda)=\int_{0}^{\infty}\left[g\left(\nu^{r}\right)-\Sigma g_{l} \nu^{l r-J r-}-H(1-\nu) g_{J} \nu^{J r}\right] \nu^{-l r-}{ }^{1} \frac{d \mu}{\alpha \beta} ;$ 
the coefficient $a_{0}(x, \lambda)$ will also involve a contribution from $k_{5}(w)$. In the integral (4.17), we observe that the integrand vanishes for small $\mu$. Hence, for small $w, H\left(1-w \mu^{-1}\right) \equiv 1$, in the region of integration. Thus, if $v^{J+1} h(v)=g(v)-\sum_{0}^{J} g_{l} v^{l}$, we may write

$$
k_{3}(w)=w^{p+r-1} \int_{0}^{\infty} h\left(w^{r} \mu^{-r}\right)\left[\varphi_{1}(\mu)-1\right] \mu^{-r-1} \frac{d \mu}{\alpha \beta},
$$

for small $w$. Thus $k_{3}(w)$ may be expanded in powers of $w^{r}$, in the same manner as (4.7).

The integral (3.22) exists for $w \neq 0$, since integration may be taken over a finite segment excluding the origin. After a change of variables,

$$
k_{4}(w)=-\frac{g_{J}}{\alpha \beta} w^{p-1} \log w
$$

Hence

$$
a_{0}^{1}(x, \lambda)=-\frac{g_{J}}{\alpha \beta}(x, \lambda)
$$

Finally, for small $w$,

$$
k_{5}(w)=w^{p-1} g_{J} \int_{0}^{\infty}\left[\varphi_{1}(\mu)-H(1-\mu)\right] \mu^{-1} \frac{d \mu}{\alpha \beta} .
$$

Combining this result with (4.20), we have

$$
a_{0}(x, \lambda)=c_{0}(x, \lambda)+g_{J}(x, \lambda) \int_{0}^{\infty} \frac{\varphi_{1}(\mu)-H(1-\mu)}{\mu} \frac{d \mu}{\alpha \beta} .
$$

This completes the proof of Lemma 4.1.

Now we may apply Theorem 2.2 to the integral (4.3). Lemma 4.1 immediately implies

Lemma 4.2. $J(x)$, given by (4.3), has an expansion in distributions homogeneous of degrees $\lambda+p, \lambda+q+\operatorname{lr}(0 \leqq l \leqq L)$, possibly including a term of the form $a_{0}^{1} c_{ \pm} x_{ \pm}^{\lambda+p} \log |x|$.

It follows that there is a similar expansion of $I(x)$, given by (4.1), provided that $g(u, v ; x, \lambda)$ can be represented as a sum of terms of the form $g(v ; x, \lambda) \varphi(u)$, plus a remainder multiplied by large powers of both $u$ and $v$. We define the second difference quotient

$$
\begin{aligned}
g_{12}(u, v ; x, \lambda) & =\int_{0}^{1} \int_{0}^{1} \frac{\partial^{2} g}{\partial u \partial v}(u s, v t ; x, \lambda) d s d t \\
& =\frac{1}{u v}[g(u, v)-g(u, o)-g(o, v)+g(o, o)] .
\end{aligned}
$$




\section{Hence}

$$
g(u, v)=g(u, o)-g(o, v)-g(o, o)+u v g_{12}(u, v) .
$$

Clearly, $g_{12}$ is smooth if $g$ is smooth. Unfortunately, the terms on the right hand side of (4.24) do not have compact support in $u$ and $v$. Although this difficulty could be circumvented by a systematic use of finite-part integrals, we prefer to work with functions with compact support.

Let $\varphi$ be a $C^{\infty}$ function with compact support, which is even, and such that $\varphi \equiv 1$ in a neighborhood of the origin. We define $h(u, v)$ by the equation

$$
\begin{aligned}
g(u, v)=g(u, o) \varphi(v) & +g(o, v) \varphi(u)-g(o, o) \varphi(u) \varphi(v) \\
& +u v h(u, s) .
\end{aligned}
$$

Using (4.24), we may write

$$
\begin{aligned}
h(u, v)= & \left(\frac{g(u, o)-g(o, o)}{u}\right)\left(\frac{1-\varphi(v)}{v}\right)+\left(\frac{g(o, v)-g(o, o)}{v}\right)\left(\frac{1-\varphi(u)}{u}\right) \\
& +g(o, o)\left(\frac{\varphi(u)-1}{u}\right)\left(\frac{\varphi(v)-1}{v}\right)+g_{12}(u, v) ;
\end{aligned}
$$

hence $h$ is a smooth function. We may apply the same process to $h(u, v)$, and thus obtain a remainder for $g$ with the factor $u^{2} v^{2}$. The process will terminate only if $g$ ceases to have the required derivatives.

We conclude that, after breaking the region of integration into quadrants, $I(x)$ may be represented as a sum of integrals of the form (4.2), plus a smooth remainder. Thus we have

THEOREM 4.2. I(x), given by (4.1), has an expansion in distributions homogeneous of degrees

$$
\lambda+\frac{\gamma+m}{\alpha}(0 \leqq m \leqq M), \lambda+\frac{\delta+l}{\beta}(0 \leqq l \leqq L),
$$

plus terms of the form $d_{ \pm} x_{ \pm}^{\sigma} \log (x)$, in case $(\gamma+m) / \alpha=(\delta+l) / \beta=\sigma$ for certain $l$ and $m$. The remainder has order greater than $\min [(\gamma+M) / \alpha),(\delta+L) / \beta)]$.

Now we shall compute the most singular term in the expansion of $I(x)$. We break the region of integration into quadrants, and evaluate the contribution from a single quadrant. The complete result would depend on the parity of $\alpha, \beta, \gamma, \delta$. As before, we write $p=\gamma / \alpha$, $q=\delta / \beta$. Observe that a lower bound on $p$ and $q$ is given by Lemma 3.1 . 
A. If $p<q$, we write, from (4.25),

$$
g(u, v)=g(o, v) \varphi(u)+[g(u, o)-g(o, o) \varphi(u)] \varphi(v)+u v h(u, v) .
$$

Since $g(u, o)-g(o, o) \varphi(u)$ is smooth and vanishes for $u=o$, the leading term arises from $g(o, v) \varphi(v)$. From Lemma 4.1, we obtain

$$
\begin{aligned}
I_{++}(x) & =\gamma(\lambda) \iint_{\substack{u>0 \\
v>0}}\left|x+u^{\alpha} v^{\beta}\right|{ }^{\lambda} u^{\gamma-1} v^{\delta-1} g(u, v ; x, \lambda) d u d v \\
& =\gamma(\lambda) \int_{0}^{\infty}|x+w|^{\lambda}\left[a_{0} w^{p-1}+o\left(w^{p-1}\right)\right] d w ;
\end{aligned}
$$

from (4.6) we have

$$
a_{0}(x, \lambda)=\int_{0}^{\infty} g(o, v ; x, \lambda) v^{\delta-\beta \gamma / \alpha} \frac{d v}{\alpha} .
$$

The leading term of $I_{++}(x)$ is given by Theorem 2.3.

B. If $q<p$, we have, similarly,

$$
\left.I_{++}(x)=\gamma(\lambda) \int_{0}^{\infty}|x+w|^{\lambda}\left[b_{0} w^{q-1}\right)+o\left(w^{q-1}\right)\right] d w,
$$

with

$$
b_{0}(x, \lambda)=\int_{0}^{\infty} g(u, o ; x, \lambda) u^{\gamma-\alpha \delta / \beta} \frac{d u}{\beta} .
$$

C. If $p=q$, we write

$$
g(u, v)=g(u, o) \varphi(v)+g(o, v) \varphi(u)-g(o, o) \varphi(u) \varphi(v)+u v h(u, v) .
$$

Applying Lemma 4.1 to each of the first three terms, we obtain

$$
I_{++}(x)=\gamma(\lambda) \int_{0}^{\infty}|x+w|^{\lambda}\left[a_{0} w^{p-1}+a_{0}^{1} w^{p-1} \log |w|+o\left(w^{p-1}\right)\right] d w,
$$

with

$$
\begin{aligned}
a_{0}(x, \lambda)= & \int_{0}^{\infty}[g(o, v)-H(1-v) g(o, o)] v^{-1} \frac{d v}{\alpha} \\
& +\int_{0}^{\infty}[g(u, o)-H(1-u) g(o, o)] u^{-1} \frac{d u}{\beta},
\end{aligned}
$$

and

$$
a_{0}^{1}(x, \lambda)=-\frac{g(o, o ; x, \lambda)}{\alpha \beta} .
$$


We remark that the preceding integrals are the finite parts of the integrals

$$
\int_{0}^{\infty} g(o, v) \frac{d v}{\alpha v} \text {, and } \int_{0}^{\infty} g(u, o) \frac{d u}{\beta u} .
$$

5. Integrals with nonvanishing Hessian. We consider integrals of the form

$$
F(x, \lambda)=\gamma(\lambda) \int_{\sigma}|f(x, u)|^{\lambda} g(x, u) d u,
$$

where $x \in X \subset R^{p}, u \in U \subset R^{n}$, and $g$ has support in the interior of the bounded set $U$. We assume that the Hessian matrix $\left[\partial^{2} f /\left(\partial u_{i} \partial u_{k}\right)\right]$ is nonsingular for all $x \in X$ and $u \in U$. In this case, a rather simple description of the singularity of $F$ can be given, using only the results of $\S 2$. Our method consists in a change of variables of integration, which enables us to write $f(x, u)=\tilde{f}(x) \pm U_{1}^{2} \pm \cdots U_{n}^{2}$. An application of Theorems 2.2 and 2.3 then shows that $F$ can be expressed in terms of $\widetilde{f}_{ \pm}^{\lambda+n / 2}$. Similar results have been obtained by a number of authors, for example J. Leray [9], L. Gårding [6], G. F. D. Duff [3], D. Ludwig [10].

Theorem 1.2 implies that the singularities of $F$ are associated with points $x_{0}, u_{0}$ where both $f\left(x_{0}, u_{0}\right)=0$ and $\left[(\partial f / \partial u)\left(x_{0}, u_{0}\right)\right]=0$. Thus we may analyse the singularity of $F$ near $x_{0}$ by covering the associated point or points $u_{0}$ by a finite collection of sufficiently small neighborhoods and choosing a partition of unity. We shall assume that this has been done. The size of the neighborhoods will be determined from the following discussion.

Since the Hessian matrix is nonsingular, we may determine $u=$ $u_{0}(X)$ from the equations $(\partial f / \partial u)(x, u)=0$ in a neighborhood of $x_{0}$. We write $u=u_{0}(x)+v, f_{1}(x, v)=f\left(x, u_{0}(x)+v\right)$. We can perform a rotation in the $v$-space so that the matrix $\left[\partial^{2} f_{1} /\left(\partial v_{i} \partial v_{k}\right)\right]$ is diagonal at $x=x_{0}, v=0$. Now we determine $\widetilde{v}_{1}\left(x, v_{2}, \cdots, v_{n}\right)$ from the equation $\partial f_{1} / \partial v_{1}=0$. Hence

$$
f_{1}(x, v)=f_{1}\left(x, \widetilde{v}_{1}, v_{2}, \cdots, v_{n}\right)+\left(v-\widetilde{v}_{1}\right)^{2} e_{1}(x, v),
$$

where $e_{1}(x, v)$ does not vanish for $x$ near $x_{0}$, if $v$ is small. Applying this process to $v_{2}, \cdots, v_{n}$ in succession, we obtain

$$
f_{1}(x, v)=f_{1}(x, 0)+\sum_{j=1}^{n}\left(v_{j}-\widetilde{v}_{j}\right)^{2} e_{j}(x, v),
$$

for $x$ near $x_{0}$, and for $v$ sufficiently small. This type of result is known as Morse's lemma (see M. Morse [11].) We set

$$
V_{j}=\left(v_{j}-\widetilde{v}_{j}\right)\left|e_{j}(x, v)\right|^{1 / 2},
$$


and

$$
\tilde{f}(x)=f_{1}(x, 0)=f\left(x, u_{0}(x)\right) .
$$

Introducing $V$ as variable of integration, we have $F$ as a sum of integrals of the form

$$
I(x, \lambda)=\gamma(\lambda) \int\left|\widetilde{f}(x) \pm V_{1}^{2} \cdots \pm V_{n}^{2}\right|^{\lambda} g_{1}(x, V) d V .
$$

We note that

$$
g_{\mathrm{s}}(x, 0)=g\left(x, u_{0}(x)\right) 2^{n / 2} \Delta^{-1 / 2},
$$

where

$$
\Delta=\left|\operatorname{det}\left(\frac{\partial^{2} f_{1}}{\partial v_{i} \partial v_{k}}\right)\right| .
$$

This integral could be handled by an application of Theorems 2.2 and $2.3 n$ times; we prefer to apply the theorems only twice. After rearrangement of indices, we may assume that

$$
e_{1}(x, v)>0, \cdots e_{k}(x, v)>0,
$$

$e_{k+1}(x, v)<0, \cdots e_{k+l}(x, v)<0$. Here $k+l=n$. We write

$$
r_{1}^{2}=V_{1}^{2}+\cdots V_{k}^{2} ; r_{2}^{2}=V_{k+l}^{2}+\cdots V_{k+l}^{2} .
$$

Then

$$
\begin{aligned}
I(x, \lambda)= & \gamma(\lambda) \iint|| \tilde{f}(x)+r_{1}^{2}-\left.r_{2}^{2}\right|^{\lambda} \\
& \times \iint g_{1}\left(x, r_{1} \omega_{1}, r_{2} \omega_{2}\right) d \omega_{1} d \omega_{2} r_{1}{ }^{k-1} r_{2}^{l-1} d r_{1} d r_{2} .
\end{aligned}
$$

Here $\omega_{1}$ and $\omega_{2}$ represent the corresponding angular variables. Integrating first over these angular variables we obtain

$$
I(x, \lambda)=\gamma(\lambda) \iint\left|\tilde{f}(x)+r_{1}^{2}-r_{2}^{2}\right|^{\lambda} g_{2}\left(x, r_{1}^{2}, r_{2}^{2}\right) r_{1}^{k-1} r_{2}^{l-1} d r_{1} d r_{2}
$$

We note that $g_{2}$ is regular in $r_{1}^{2}$ and $r_{2}^{2}$, and

$$
g_{2}(x, 0,0)=\frac{4(2 \pi)^{n / 2}}{\Gamma\left(\frac{k}{2}\right) \Gamma\left(\frac{l}{2}\right)} \Delta^{-1 / 2} g\left(x, u_{o}(x)\right) \text {. }
$$

Now we may expand $g_{2}$ in integral powers of $r_{1}^{2}$ and $r_{2}^{2}$; for fixed $\lambda$ the remainder will be smooth in $x$ if enough terms are taken. It therefore suffices to find the singularity of a single term of the form 


$$
J(x, \lambda)=\gamma(\lambda) \iint_{s_{s_{2} \geq 0} \geq 0}\left|\tilde{f}(x)+s_{1}-s_{2}\right|^{\lambda} s_{1}^{k / 2-1} s_{2}{ }^{l / 2-1} d s_{1} d s_{2} .
$$

The leading term of $I(x, \lambda)$ will have precisely the form (5.3), multiplied by

$$
\frac{(2 \pi)^{n / 2}}{\Gamma\left(\frac{k}{2}\right) \Gamma\left(\frac{l}{2}\right)} \Delta^{-1 / 2} g\left(x, u_{0}(x)\right) .
$$

Now applying Theorem 2.3, we see that

$$
\begin{aligned}
J(x, \lambda)= & \gamma(\lambda) \Gamma\left(\frac{l}{2}\right) \Gamma(\lambda+1)\left[\frac{\sin \pi\left(\lambda+\frac{l}{2}\right)-\sin \pi \frac{l}{2}}{\sin \pi\left(\lambda+\frac{l}{2}\right)} I_{+}\right. \\
& \left.+\frac{\sin \pi \lambda}{\sin \pi\left(\lambda+\frac{l}{2}\right)} I_{-}\right]+R(x, \lambda),
\end{aligned}
$$

where

$$
I_{ \pm}=\frac{1}{\Gamma\left(\lambda+\frac{l}{2}+1\right)} \int_{s_{1}>0}\left(\tilde{f}(x)+s_{1}\right)_{ \pm}{ }^{\lambda+l / 2} s_{1}^{k / 2-1} d s_{1},
$$

and $R(x, \lambda)$ is regular. Now applying Theorem 2.2 to $I_{ \pm}$, we find that

$$
\begin{aligned}
J(x, \lambda)= & \Gamma\left(\frac{l}{2}\right) \Gamma\left(\frac{k}{2}\right) \gamma(\lambda) \Gamma(\lambda+1) \\
& \times\left[\frac{\sin \pi\left(\lambda+\frac{l}{2}\right)-\sin \pi \frac{l}{2}}{\sin \pi\left(\lambda+\frac{n}{2}\right)} \frac{\tilde{f}_{+}^{\lambda+(n / 2)}}{\Gamma\left(\lambda+\frac{n}{2}+1\right)}\right. \\
& \left.+\frac{\sin \pi\left(\lambda+\frac{k}{2}\right)-\sin \pi \frac{k}{2}}{\sin \pi\left(\lambda+\frac{n}{2}\right)} \frac{\tilde{f}_{-}^{\lambda+(n / 2)}}{\Gamma\left(\lambda+\frac{n}{2}+1\right)}\right]+R_{2}(x, \lambda) .
\end{aligned}
$$

Hence the leading term of $I(x, \lambda)$ is given by

(5.4) $\quad(2 \pi)^{n / 2} \Delta^{-1 / 2} g\left(x, u_{0}(x)\right)$

$$
\times\left[d_{+} \frac{\tilde{f}_{+}{ }^{\lambda+n / 2}}{\Gamma\left(\lambda+\frac{n}{2}+1\right)}+d_{-} \frac{\tilde{f}_{-}{ }^{\lambda+n / 2}}{\Gamma\left(\lambda+\frac{n}{2}+1\right)}\right],
$$

with 


$$
\begin{aligned}
& d_{+}=\gamma(\lambda) \Gamma(\lambda+1)\left[\frac{\sin \pi\left(\lambda+\frac{l}{2}\right)-\sin \pi \frac{l}{2}}{\sin \pi\left(\lambda+\frac{n}{2}\right)}\right], \\
& d_{-}=\gamma(\lambda) \Gamma(\lambda+1)\left[\frac{\sin \pi\left(\lambda+\frac{k}{2}\right)-\sin \pi \frac{k}{2}}{\sin \pi\left(\lambda+\frac{n}{2}\right)}\right] .
\end{aligned}
$$

The coefficients $d_{ \pm}$have simple poles as functions of $\lambda$ according to the following scheme:

If $k$ and $l$ are both even, there are poles if $\lambda$ is of the form $-2 q, q$ integer $\geqq 0$.

If $h$ and $l$ are both odd, there are poles if $\lambda=-2 q-1, q$ integer $\geqq 0$.

If $k+l$ is odd, there are poles if $\lambda=q+1 / 2, q$ any integer.

Since $I(x, \lambda)$ is regular for all $\lambda$, of course the sum of the residues at these poles is zero.

\section{BIBLIOGRAPHY}

1. G. A. Bliss, Fundamental existence theorems, New York, 1913.

2. R. Courant, Methods of mathematical physics, II, New York, 1962.

3. G. F. D. Duff, On the Riemann matrix of a hyperbolic system, MRC Tech. Rep. \#246, U. of Wisconsin, 1961 .

4. A. Erdélyi, Asymptotic Expansions, New York, 1956.

5. J. Focke, Asymptotische Entwicklungen mittels der methode der stationären phase; Berichte Säch. Akad. der Wiss. Leipzig, V. 101, Heft 3, 1954.

6. L. Gårding, Transformation de Fourier des distributions homogènes, Bull. Sci. Math. France (89), 4, (1961), 381-428.

7. I. M. Gelfand and G. E. Shilov, Verallgemeinerte Funttionen, I. Berlin, 1960.

8. D. S. Jones and M. Kline, Asymptotic expansion of multiple integrals and the method of stationary phase, J. Math. Physics 37 (1958) 1-28.

9. J. Leray, Le calcul différentiel et intégral sur une Variété analytique complexe, Bull. Soc. Math. France 87 (1959) 6-180.

10. D. Ludwig, The singularities of the Riemann function, Rep. No. NYO-9351, Inst. of Math. Sci. New York U. 1961.

11. M. Morse, The Calculus of variations in the large, Providence, 1934.

12. R. J. Walker, Algebraic curves, Princeton, 1950.

Courant Institute of Mathematical Sciences, NEW YORK UNIVERSITY 



\section{PACIFIC JOURNAL OF MATHEMATICS}

\section{EDITORS}

H. Samelson

Stanford University

Stanford, California

R. M. Blumenthal

University of Washington

Seattle, Washington 98105
J. Dugundu

University of Southern California Los Angeles, California 90007

*Richard Arens

University of California

Los Angeles, California 90024

\section{ASSOCIATE EDITORS}
E. F. BECKENBACH
B. H. NeumanN
F. WOLF
K. YOSIDA

\section{SUPPORTING INSTITUTIONS}

UNIVERSITY OF BRITISH COLUMBIA CALIFORNIA INSTITUTE OF TECHNOLOGY UNIVERSITY OF CALIFORNIA MONTANA STATE UNIVERSITY

UNIVERSITY OF NEVADA

NEW MEXICO STATE UNIVERSITY

OREGON STATE UNIVERSITY

UNIVERSITY OF OREGON

OSAKA UNIVERSITY

UNIVERSITY OF SOUTHERN CALIFORNIA
STANFORD UNIVERSITY

UNIVERSITY OF TOKYO

UNIVERSITY OF UTAH

WASHINGTON STATE UNIVERSITY

UNIVERSITY OF WASHINGTON

AMERICAN MATHEMATICAL SOCIETY CALIFORNIA RESEARCH CORPORATION SPACE TECHNOLOGY LABORATORIES NAVAL ORDNANCE TEST STATION 


\section{Pacific Journal of Mathematics}

\section{Vol. 15, No. $1 \quad$ September, 1965}

Donald Charles Benson, Unimodular solutions of infinite systems of linear

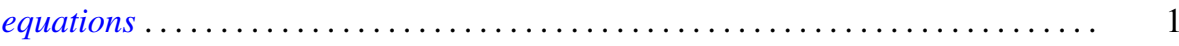

Richard Earl Block, Transitive groups of collineations on certain designs . . . . . . 13

Barry William Boehm, Existence of best rational Tchebycheff approximations .... . 19

Joseph Patrick Brannen, A note on Hausdorff's summation methods . . . . . . . . . . 29

Dennison Robert Brown, Topological semilattices on the two-cell ............ 35

Peter Southcott Bullen, Some inequalities for symmetric means . . . . . . . . . . 47

David Geoffrey Cantor, On arithmetic properties of coefficients of rational

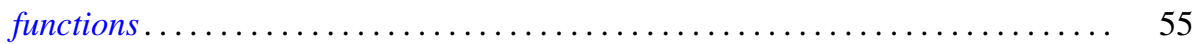

Luther Elic Claborn, Dedekind domains and rings of quotients . . . . . . . . . 59

Allan Clark, Homotopy commutativity and the Moore spectral sequence ........ 65

Allen Devinatz, The asymptotic nature of the solutions of certain linear systems of

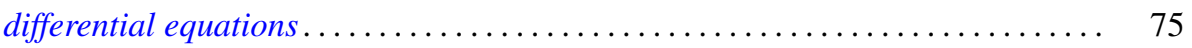

Robert E. Edwards, Approximation by convolutions ................... 85

Theodore William Gamelin, Decomposition theorems for Fredholm operators . . . . . 97

Edmond E. Granirer, On the invariant mean on topological semigroups and on

topological groups .................................. 107

Noel Justin Hicks, Closed vector fields . . . . . . . . . . . . . . . . . . . 141

Charles Ray Hobby and Ronald Pyke, Doubly stochastic operators obtained from

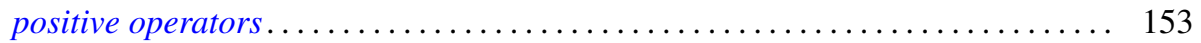

Robert Franklin Jolly, Concerning periodic subadditive functions . . . . . . . . . 159

Tosio Kato, Wave operators and unitary equivalence . . . . . . . . . . . . . . 171

Paul Katz and Ernst Gabor Straus, Infinite sums in algebraic structures . . . . . . . 181

Herbert Frederick Kreimer, Jr., On an extension of the Picard-Vessiot theory ...... 191

Radha Govinda Laha and Eugene Lukacs, On a linear form whose distribution is

identical with that of a monomial ......................... 207

Donald A. Ludwig, Singularities of superpositions of distributions . . . . . . . . . 215

Albert W. Marshall and Ingram Olkin, Norms and inequalities for condition

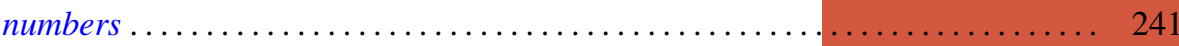

Horace Yomishi Mochizuki, Finitistic global dimension for rings . . . . . . . . . . 249

Robert Harvey Oehmke and Reuben Sandler, The collineation groups of division

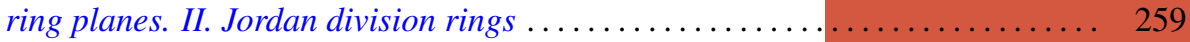

George H. Orland, On non-convex polyhedral surfaces in $E^{3} \ldots \ldots \ldots \ldots \ldots \ldots \ldots 267$

Theodore G. Ostrom, Collineation groups of semi-translation planes . . . . . . . . 273

Arthur Argyle Sagle, On anti-commutative algebras and general Lie triple

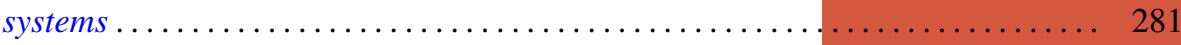

Laurent Siebenmann, A characterization of free projective planes . . . . . . . . . 293

Edward Silverman, Simple areas.................................. 299

James McLean Sloss, Chebyshev approximation to zero .................. 305

Robert S. Strichartz, Isometric isomorphisms of measure algebras . . . . . . . . . 315

Richard Joseph Turyn, Character sums and difference sets . . . . . . . . . . . . 319

L. E. Ward, Concerning Koch's theorem on the existence of arcs . . . . . . . . . . 347

Israel Zuckerman, A new measure of a partial differential field extension ......... 357 